\section{Effect of silver nanoparticles on hardness in medium-density fiberboard (MDF)}

\author{
Hamid Reza Taghiyari ${ }^{(1)}$, Jack Norton ${ }^{(2)}$
}

\begin{abstract}
Effect of silver nanoparticles on hardness in medium-density fiberboard (MDF) was studied here. A $400 \mathrm{ppm}$ aqueous nanosilver suspension was used at three consumption levels of 100,150 , and $200 \mathrm{~mL} \mathrm{~kg}^{-1}$, based on the dry weight of wood fibers; the results were then compared with the control panels. The size range of silver nanoparticles was $30-80 \mathrm{~nm}$. Composite mats were hot-pressed for 6,8 , and $10 \mathrm{~min}$. Results showed that the uniform and even dispersion of nanoparticles throughout the MDF-matrix significantly contributed to an increase in the hardness at lower hot-press time of $6 \mathrm{~min}$. In the longer hot-press times, however, over-heating of the mat resulted in significant a decrease of hardness values. Significant high correlation was observed between water absorption and thickness swelling.
\end{abstract}

Keywords: Composite Board, Heat Transferring Property, Metal Nanoparticles, Nanosilver, Thermal Conductivity Coefficient, Wood Fiber

\section{Introduction}

The harvested wood of fast-growing trees is not usually suitable for the furniture industry; however, they provide a sustainable source for paper and composite-manufacturing industries, and natural regeneration would help the process (Ruprecht et al. 2012, Maresi et al. 2013). Wood-composite panels offer the advantage of a homogeneous structure which may be important for many design purposes (Valenzuela et al. 2012). Due to the low thermal conductivity coefficient of wood (Taghiyari et al. 2013a), some studies have so far been carried out to decrease the maximum pressing time or in crease the heat-transferring rate to the core section of the mat (Lehmann et al. 1973, Taghiyari et al. 2013b). Hot press time is dependant on the thickness of the composite mat, press temperature, closing rate, and most importantly, moisture distribution throughout the mat (Doost-Hoseini et al. 2013, Taghiyari et al. 2013b). There is a limit to moisture content of the mat because of two main reasons: (i) increase in the hotpress time; and (ii) occurrence of blows in wood-composite panels. A blow occurs

when excess moisture in the board explosively evaporates at temperatures greater than $100{ }^{\circ} \mathrm{C}$, once pressure is released. Furthermore, for urea-formaldehyde (UF) resin, there is a limitation of MC level (Papadopoulos 2007, Stockel et al. 2012). Therefore, rapid transfer of heat to the core section of the mat, and polymerization of resin, has always been a challenge.

Many areas of science have used nanotechnology to enhance the effectiveness of materials at nano scales. Heat-transferring property of metal nanoparticles (Khojier et al. 2012) and mineral nanofibers (Haghighi Poshtiri et al. 2013) was reported to improve some properties in solid wood as well as wood-composite materials. However, little study has so far been carried out to practically investigate the effects of different nano-materials in wood-composite panels. The present research project was therefore conducted to study the effects of silver nanoparticles as well as different hot-press times on the hardness of medium-density fiberboards. The relations between the hardness and some physical properties of the panels were investigated as well.

(1) Wood Science \& Technology Department, Faculty of Civil Engineering, Shahid Rajaee Teacher Training University, Tehran (Iran); (2) Retired, Horticulture \& Forestry Science, Queensland Department of Agriculture, Forestry and Fisheries (Australia)

@ Hamid Reza Taghiyari (htaghiyari@yahoo.com)

Received: Nov 27, 2013 - Accepted: Aug 07, 2014

Citation: Taghiyari HR, Norton J, 2014. Effect of silver nanoparticles on hardness in medium-density fiberboard (MDF). iForest 8: 677-680 [online 2014-12-17] URL: http://www. sisef.it/iforest/contents/?id=ifor1188-007

Communicated by: Giacomo Goli

\section{Materials and methods}

\section{Specimen preparation}

Wood fibers were obtained from the Sanaye Choobe Khazar Company (MDF Caspian Khazar, Amol, Iran). The fibers were a mixture of beech, alder, maple, hornbeam, and poplar from forests of the Gillan province (Amol, Iran). The raw materials were free from fungi deterioration. This material was used to prepare boards that were $16 \mathrm{~mm}$ thick and had a density of $0.68 \mathrm{~g} \mathrm{~cm}^{-3}$. A laboratory hot press produced by the Mehrabadi Machinery Mfg. Co. (Tehran, Iran) was used to prepare test specimens. The size of the hot plates was $50 \times 50 \mathrm{~cm}$. The total nominal pressure applied by the hot plates was 160 bars at a fixed plate temperature of 150 ${ }^{\circ} \mathrm{C}$. Hot-pressing continued for 6,8 , and 10 minutes. Urea-Formaldehyde resin (UF) was supplied from Pars Chemical Industries Company (Tehran, Iran). Based on the dry weight of wood fibers, UF resin $200-400 \mathrm{cP}$ in viscosity, 47 seconds of gel time, and $1277 \mathrm{~g} \mathrm{~cm}^{-3}$ in density was used at a rate of $10 \%$ in the composite panels. Specimens were kept in a conditioning chamber $(20 \pm 2$ ${ }^{\circ} \mathrm{C}$, and $65 \pm 2 \%$ relative humidity) for four weeks before testing. The moisture content of the specimens at the time of testing was $7.5 \%$ because wood has a thermo-hygromechanical behavior and its properties depends on the combined action of temperature, relative humidity, and mechanical load variations (Figueroa et al. 2012). Five boards were made for each treatment group.

\section{Nano-silver application}

A 400-ppm aqueous suspension of nanosilver (NS) was produced and applied to specimens using an electrochemical technique. The nano-suspension was prepared by transferring the silver metal ion from the aqueous phase to the organic phase, where it reacted with a monomer. The formation and size of the silver nanoparticles was monitored by transmission electron microscopy (TEM). Samples for TEM were prepared by drop-coating the Ag nanoparticle suspensions on to carbon-coated copper grids. Micrographs were obtained using an EM-900 ZEISS transmission electron microscope. The size range of silver nanoparticles was $30-80 \mathrm{~nm}$. The $\mathrm{pH}$ of the suspension was 67. Two kinds of surfactants (anionic and cationic) were used in the suspension as stabilizer; the concentration of the surfactants was two times the nano-silver particles. The nano-suspension was applied at three consumption levels, including nanosilver 100 (NS-100, $100 \mathrm{~mL} \mathrm{~kg}^{-1}$ ), nanosilver 150 (NS$150,150 \mathrm{~mL} \mathrm{~kg}^{-1}$ ), and nanosilver 200 (NS$200,200 \mathrm{~mL} \mathrm{~kg}^{-1}$ ). After impregnating the wood specimens with the silver nano-suspension, SEM micrographs showed uniform 


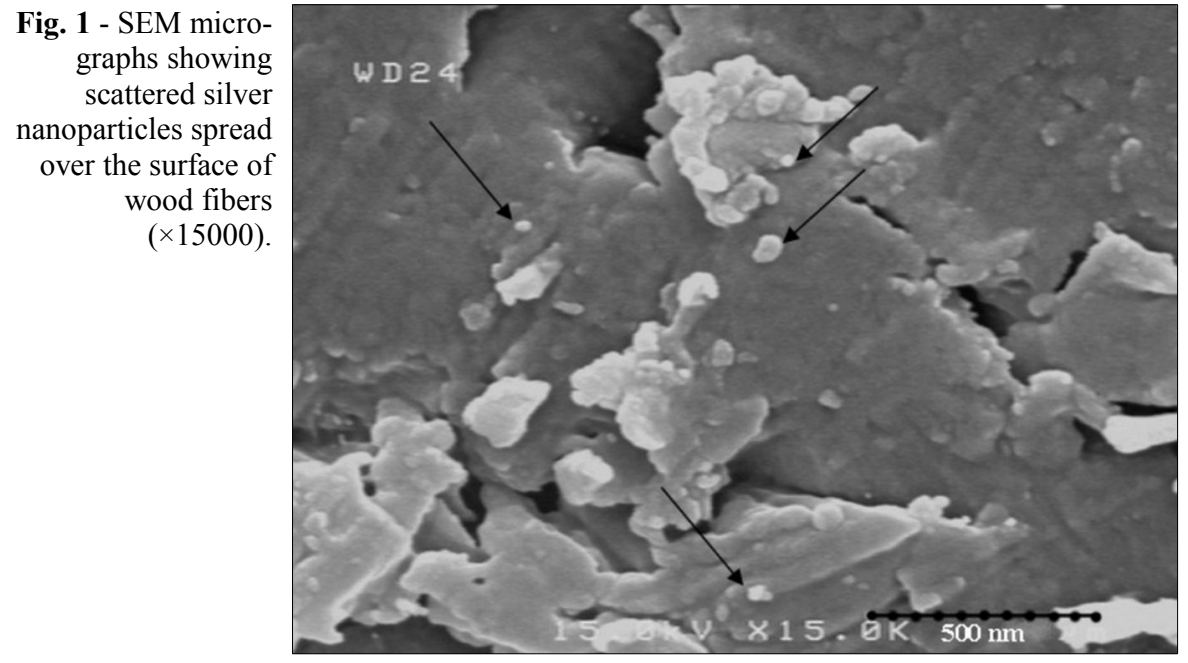

dispersion of nanoparticles on wood fibers (Fig. 1). After preparation, the nano-suspension was mixed with the UF resin and sprayed on the fibers.

\section{SEM imaging}

SEM imaging was done at thin-film laboratory, FE-SEM lab (Field Emission), School of Electrical \& Computer Engineering, The University of Tehran (Iran); a field-emission cathode in the electron gun of a scanning electron microscope provided narrower probing beams at low as well as high electron energy, resulting in both improved spatial resolution and minimized sample charging and damage.

Fig. 2 - Hardness in the nanosilvertreated medium-density fiberboard at the penetration depths of $2 \mathrm{~mm}$ (A) and 5.4 $\mathrm{mm}(\mathrm{B})(\mathrm{NS}=$ nanosilver; $\mathrm{HP}=$ hot-press time).
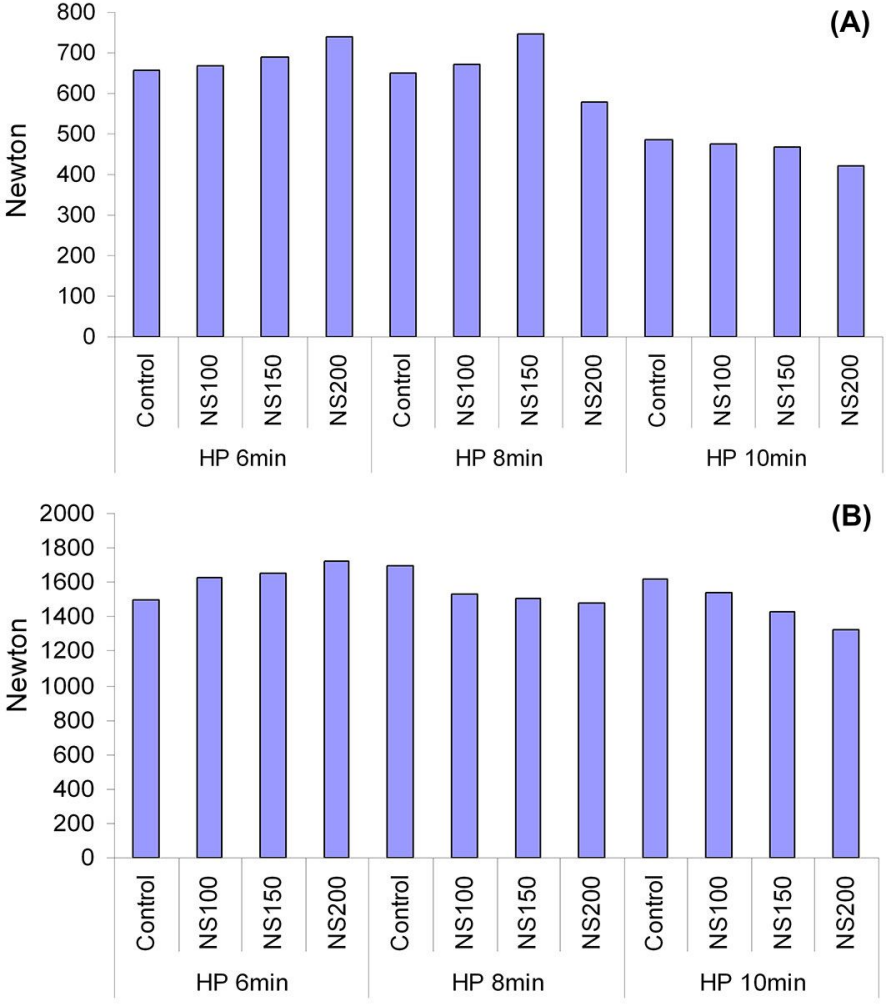

Hardness was measured at five different penetration depths of the hardness ball, that is, hardness loading was measured at 2, 3, 4, 5, and $5.4 \mathrm{~mm}$ of the penetration of the ball into the composite-board specimens.

\section{Water absorption and thickness swelling}

Physical properties of water absorption (WA) and thickness swelling (TS) were also carried out in accordance with Iranian Standard ISIRI 9044 PB Type P2 (compatible with ASTM D1037-99 specifications). From each panel, three specimens were prepared. Specimens were immersed in distilled water for 2 and 24 hours. WA and TS were then measured and expressed as a percent for each specimen. The size of specimens was $150 \times 150 \mathrm{~mm}$. Specimens were conditioned prior to the tests. For water absorption, a digital scale with $0.01 \mathrm{~g}$ precision was used for weighing specimens before and after immersion in water. For thickness swelling, a digital micrometer with $0.01 \mathrm{~mm}$ precision was used to measure five points; the five points included one in the center, and four points at each midway side of the specimens. The average of five points was reported.

\section{Statistical analysis}

Statistical analysis was conducted using SAS software, version 9.2 (2010). Two-way analysis of variance (ANOVA) was performed on the data to determine significant differences at the $95 \%$ level of confidence. Hierarchical cluster analysis, including dendrograms and Ward's methods with squared Euclidean distance intervals, was carried out using SPSS/18 (2010). Cluster analysis was performed to find similarities and dissimilarities between treatments based on more than one property simultaneously. The scaled indicator in each cluster analysis shows how much treatments are similar or different; lower scale numbers show more similarities while higher ones show dissimilarities. Fitted-line and scatter plots were made by Minitab software, version 16.2.2 (2010).

\section{Results and discussion}

SEM images showed uniform spread of silver nanoparticles over the surface of wood fibers in the wood-composite matrix, facilitating easier transfer of heat from the hotplates of the press to the wood-matrix, as reported by Taghiyari et al. (2013b - Fig. 1).

Results of the hardness tests showed significant difference in the hardness values measured at different depths of penetration of the ball. As the depth of penetration increased, the hardness increased too (Fig. 2A, Fig. $2 \mathrm{~B})$. The maximum hardness values at the depths of 2 and $5.4 \mathrm{~mm}$ were found in NS150-HP8 (748 N) and NS200-HP6 (1719 N) treatments, respectively; that is, increase in the depth of penetration from 2 to $5.4 \mathrm{~mm}$ 
resulted in a difference in hardness up to $130 \%$. Hardness values of the two press times of 6 and 10 min showed similar decreasing or increasing trends at the two depths of penetration. However, different trends were observed when press time was fixed at $8 \mathrm{~min}$. This showed the effect of facilitated heat-transfer to the mat due to silver nanoparticles. However, it should be noticed that as the depth of penetration increased, the diameter of the Janka modified ball increased too. Further studies should be carried out with equal conditions to evaluate absolute hardness at different depths of penetration.

Increase in the press time resulted in a significant decrease in hardness at the depth of $2 \mathrm{~mm}$. At the higher penetration depth of 5.4 $\mathrm{mm}$, though, the fluctuations depended on the treatment. The reason for the decrease in hardness at the lower depth of $2 \mathrm{~mm}$ may be attributed to the de-polymerization of the resin due to the extreme absorption of temperature from the hot-press plates. However, more direct evidence on the hypothesis of de-polymerization of resin is needed to come to a final explanation. That is, heat was easily transferred from the hot-plates to the surface layer of MDF at the depth of 2-3 mm (Taghiyari et al. 2013b); when the duration of hot-press was $6 \mathrm{~min}$, the temperature transferred to the MDF-mat was mainly used to polymerize the UF-resin. Increasing the hot-press time to $10 \mathrm{~min}$, the polymerized resin was over-heated, and de-polymerization of the UF-resin gradually occurred. Easier transfer of heat to the core section of the mat was experimentally approved (Taghiyari et al. 2013b). However, other effects such as the catalytic properties of silver nanoparticles on the curing time of resin should also be investigated in details in further studies. Nearly the same trend was observed in the NS-treated panels, though silver nanoparticles intensified the process of de-polymerization.

The high thermal conductivity coefficient of silver nanoparticles, smoothly scattered throughout the fibers (Fig. 1), facilitated the transfer of heat to the MDF-mat. At lower hot-press time of $6 \mathrm{~min}$, the facilitated transfer of heat to the mat resulted in a better polymerization of the UF-resin; consequently, the hardness values significantly increased (Fig. 2). At higher hot-press time of $10 \mathrm{~min}$ though, the extra heat was used in the depolymerization of the resin, ultimately decreasing the hardness. A steady increase in the hardness was observed as the content of nanosilver gradually increased to its up-most limit of $200 \mathrm{~mL} \mathrm{~kg}^{-1}$ in the lower hot-press time (6 min), showing better polymerization of the resin due to the facilitated heat-transfer. Nearly the same trends were observed at different depths of penetration in the hotpress times of 6 and $10 \mathrm{~min}$. The opposite

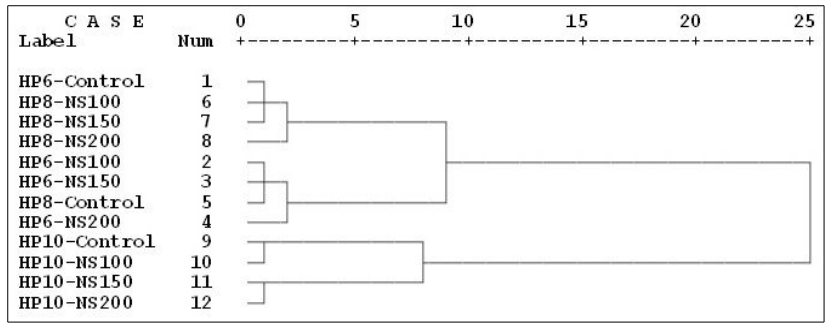

Fig. 3 - Cluster analysis of the twelve treatments of medium-density fiberboards based on the hardness values measured at five different depths of penetration $(2,3,4,5$, and $5.4 \mathrm{~mm}$ ) into the MDF.

was observed in the longer hot-press time of $10 \mathrm{~min}$; that is, the facilitated heat-transfer by the silver nanoparticles was used in the de-polymerization of the resin, resulting in a decreasing-trend in the hardness values as the NS-content increased.

Cluster analysis of the twelve treatments based on the hardness values measured at the five depths of penetrations $(2,3,4,5$, and $5.4 \mathrm{~mm}$ ) indicated that all HP-10 treatments were significantly categorized in a different cluster (Fig. 3). This proved the significant effect of hot-press time on the overall hardness in medium-density fiber-boards. In this connection, the two HP-10 treatments with higher NS-content (NS-150 and NS-200) were also clustered differently in comparison to the two treatments with lower NS-content (control and NS-100 treatments); this also indicated the significant effect of the improvement in thermal conductivity of the MDFmat on the hardness. As to the treatments with 6 min hot-press time, all HP-6 treat- ments with NS-content were closely clustered to the HP-8 control treatment. This demonstrated that the improvement in the thermal conductivity of the mat by nanosilver improved the hardness in a way that it compensated for the two extra minutes of hot-press in HP8-control treatment, that is, the increase in the hot-press from 6 to $8 \mathrm{~min}$. This would be very important for industry, because hot-press time is a bottle-neck to production in the wood-composite manufacturing factories and a small decrease in the hot-press time, can have a significant economic impact.

High significant correlation $\left(\mathrm{R}^{2}=0.912\right)$ was found between water absorption (the amount of water absorbed by specimens after 2-hour immersion in distilled water) and thickness swelling (the amount of swelling that happens after 2-hour immersion in distilled water - Fig. 4). Similar significant correlations were also reported in particleboards as a result of silver and copper nanoparticles.
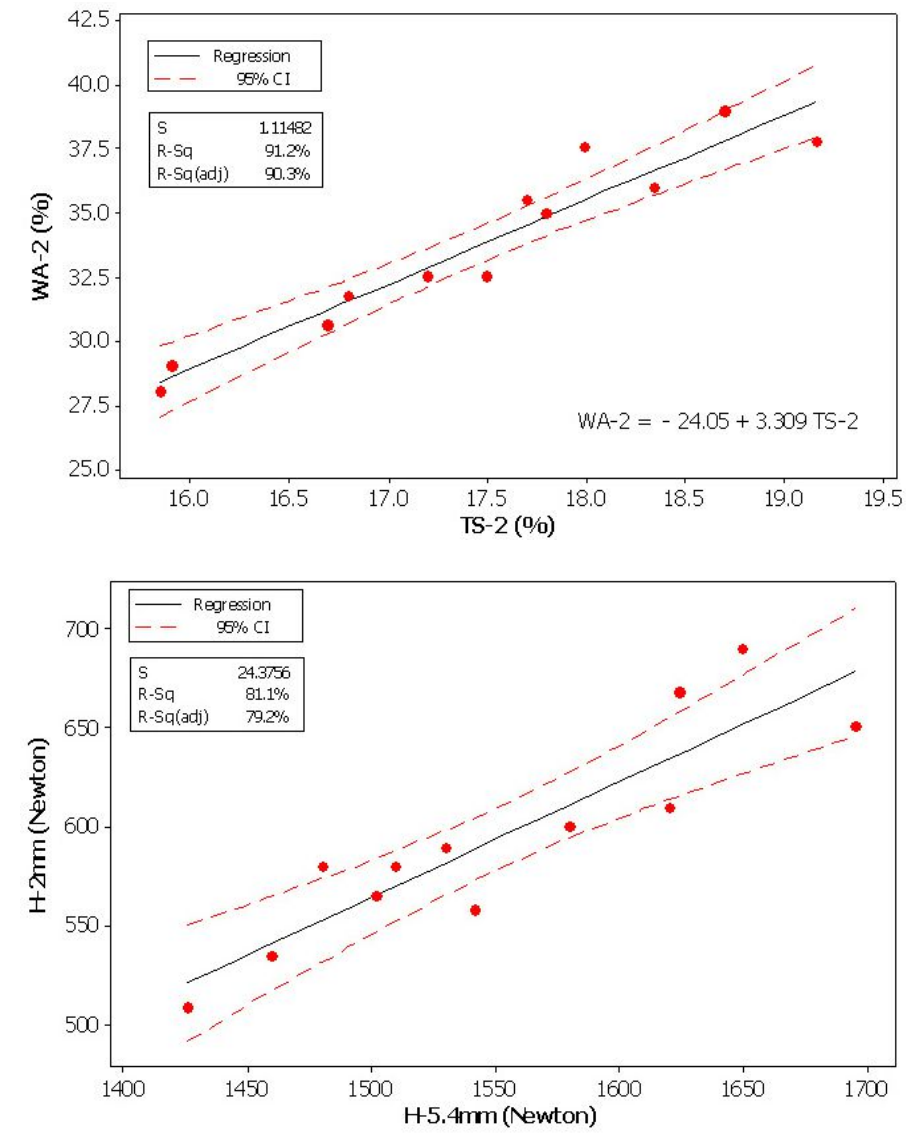

Fig. 4 - Fitted line plot between water absorption (WA) versus thickness swelling (TS) values after 2 hours of immersion in distilled water $(\%)$.

Fig. 5 - Fitted line plot between hardness values measured at two depths of penetration of 2 $\mathrm{mm}(\mathrm{H}-2 \mathrm{~mm})$ vs. 5.4 $\mathrm{mm}$ (H-5.4mm) (Newton). 
In fact, the improved thermal conductivity resulted in a significant higher rate of heattransfer to the core section of the mat (Taghiyari et al. 2013a), consequently, the resin was better and more efficiently cured and ultimately, the physical and mechanical properties were significantly improved. The fit ted-line plot between the hardness values measured at different depths of penetration showed a high significant correlation $\left(\mathrm{R}^{2}=\right.$ 0.81 - Fig. 5). However, it was significantly lower than what was found between WA and TS. This can be related to the de-polymerization of resin in the surface layers of the NStreated panels. In fact, NS-treated panels showed an increasing trend in hardness values at 6 min press time (Fig. 2A); however, the trend was decreasing when press time increased to $10 \mathrm{~min}$. This primarily strengthened the hypothesis of de-polymerization of resin, though it also explained why correlation in hardness values at different depths of penetration was not as high as in the physical properties.

The optimum consumption level for metal nanoparticles was reported to be dependent on many factors, including the hot press temperature, hot-press duration, thermal conductivity coefficient of metal or mineral nanoparticles, moisture content of the mat, and the type and density of composite panels (Taghiyari et al. 2013b). Based on the results obtained in the present study, it was concluded that by proper trade-off between nanosilver-content and press-time, two major objectives at wood-composite manufacturing factories can be obtained, including the improvement of hardness and physical properties of water absorption and thickness swelling, as well as the decrease in the hotpress time.

\section{Conclusions}

Effects of a 400 ppm aqueous suspension of silver nanoparticles, as well as hot-press time on the hardness values in medium-density fiberboards (MDF) were studied. Correlations between water absorption and thickness swelling was also calculated and inter- preted. MDF-mat was hot-pressed for 6, 8, and 10 minutes. The obtained results showed a significant effect of both factors of hotpress duration and heat-transferring property of silver nanoparticles on the hardness values. Based on our results, it was concluded that heat-transferring property of silver nanoparticles had an increasing effect on hardness. However, extension of hot-press time to more than 6 min resulted in a decrease in hardness which can be attributed to the depolymerization of the UF-resin. Significant high correlation was observed between water absorption and thickness swelling.

\section{Acknowledgments}

The authors are grateful to Mr. Majid Ghazizadeh, the internal sales manager of Pars Chemical Industries Company (Tehran, Iran), for the procurement of the resin for the present study. We also appreciate the kind cooperation of Engr. Asghar Jahangiri and Pezhman Nouri (experts in wood-composite materials) in the interpretation of results.

\section{References}

Doost-Hoseini K, Taghiyari HR, Elyasi A (2013). Correlation between sound absorption coefficients with physical and mechanical properties of insulation boards made from sugar cane bagasse. Journal of Composites Part B 58: 1015. - doi: 10.1016/j.compositesb.2013.10.011

Figueroa M, Bustos C, Dechent P, Reyes L, Cloutier A, Giuliano M (2012). Analysis of rheological and thermo-hygro-mechanical behaviour of stress-laminated timber bridge deck in variable environmental conditions. Maderas Ciencia y Tecnologia 14 (3): 303-319. [online] URL: http://revistamaderas.cl/ojs/index.php/remaderas/ article/viewArticle $/ 486$

Haghighi Poshtiri A, Taghiyari HR, Karimi AN (2013). The optimum level of nano-wollastonite consumption as fire-retardant in poplar wood (Populus nigra). International Journal of Nano Dimension 4 (2): 141-151. [online] URL: http:// www.ijnd.ir/?_action $=$ articleInfo\&article $=3696$ $\&$ vol $=770$

Khojier K, Zolghadr S, Zare N (2012). Structural, electrical, and optical properties of molybdenum oxide thin films prepared by post-annealing of Mo thin films. International Journal of Bio-Inorganic Hybrid Nanomaterials 1 (3): 199-207.

Lehmann WF, Geimer RL, Hefty FV (1973). Factors affecting particleboard pressing time: interaction with catalyst systems. Research Paper FPL 208, USDA Forest Service, Madison, Wisconsin, USA, pp. 22.

Maresi G, Oliveira Longa CM, Turchetti T (2013). Brown rot on nuts of Castanea sativa Mill: an emerging disease and its causal agent. iForest - Biogeosciences and Forestry 6 (5): 294 301. - doi: 10.3832/ifor0952-006

Papadopoulos AN (2007). Property comparisons and bonding efficiency of UF and PMDI bonded particleboards as affected by key process variables. BioResources 1 (12): 201-208. [online] URL: http://ojs.cnr.ncsu.edu/index.php/BioRes/ article/view/BioRes_01_2_201_208_Papadopou los_Property_Comoparisons_Bonding

Ruprecht H, Vacik H, Steiner H, Frank G (2012). ELENA - a methodological approach for the long term monitoring of the natural regeneration of natural forest reserves dominated by Norway spruce (Vaccinio piceetea). Austrian Journal of Forest Science 129 (2): 67-105.

Stockel F, Konnerth J, Moser J, Kantner W, Gindl-Altmutter W (2012). Micromechanical properties of the interphase in pMDI and UF lines. Wood Science and Technology 46 (4): 611-620. - doi: 10.1007/s00226-011-0432-0

Taghiyari HR, Mobini K, Sarvari Samadi Y, Doosti Z, Karimi F, Asghari M, Jahangiri A, Nouri P (2013a). Effects of nano-wollastonite on thermal conductivity coefficient of medium-density fiberboard. Journal of Nanomaterials and Molecular Nanotechnology 2: 1-1. - doi: 10.41 72/2324-8777.1000106

Taghiyari HR, Moradiyan A, Farazi A (2013b). Effect of nanosilver on the rate of heat transfer to the core of the medium density fiberboard mat. International Journal of Bio-Inorganic Hybrid Nanomaterials 2 (1): 303-308.

Valenzuela J, Leyser E, Pizzi A, Westermeyer C, Gorrini B (2012). Industrial production of pine tannin-bonded particleboard and MDF. European Journal of Wood and Wood Products 70: 735-740. - doi: 10.1007/s00107-012-0610-2 